\title{
POLYPS OF THE COLON BEYOND THE REACH OF THE SIGMOIDOSCOPE*
}

\author{
BY \\ JEAN DUHAMEL and PIERRE BAUCHE \\ From Hôpitaux de Paris, France, and Université de Liège, Belgium
}

Amongst 350 children treated for recto-colonic polyps at the Hôpital des Enfants-Malades, we have seen 11 cases of polyps of the colon out of reach of the sigmoidoscope (remote polyps). A further 4 cases from the Hôpital de Bavière de Liège (Belgium) have been added, making in all 15 out of a total of 400 cases of recto-colonic polyps.

Are remote polyps in children much less common than recto-sigmoidal polyps? In the adult, this disproportion is only apparent, systematic necropsies showing clearly that the localization of adenomas in the different segments of the large intestine (rectum included) is fairly regular (Chapman, 1963; Feyrter, 1931) (Fig. 1a).

The studies on adults show that the frequency increases with age, with $20 \%$ in the third and the fourth decades, $31 \%$ in the fifth, $61 \%$ in the sixth, and more than $75 \%$ after 80 years. Children's polyps are quite different. Helwig (1946) found that amongst 449 necropsies of children and young adults under 21 years of age, there were only 5 subjects suffering from polyps: 1 polyp of the rectum in a 4-year-old boy; 8 polyps of the rectum in an 8 -year-old boy; 1 polyp of the rectum in a 12-year-old girl; 1 polyp of the ascending colon in a 7-year-old girl; and 1 polyp of the caecum in a 15-year-old girl. In all, there were 10 polyps of the rectum, 1 of the ascending colon, and 1 of the caecum (Fig. 1b). The incidence must be higher in small children on account of the extreme scarcity of these polyps in adolescents. It is well known that they are quite exceptional before the age of 1 year; their frequency increases very quickly after 2 years, to reach a maximum between 4 and 5 years (Duhamel, 1958). After this age, polyps become uncommon (possibly on account of self-destruction), and are very rare after 14 years. Between 15 and 20, adenomas are uncommon, though we have personally observed 5 cases. During the period that we shall call the 'adolescent gap', our observations have shown the persistence of infantile polyps after the age

* A paper read at a meeting of the British Association of Paediatric Surgeons in Rotterdam, September 1964. of 15. From this fact some very important problems emerge concerning the fate of polyps of childhood and the relation between these and adult polyps. This persistence into adolescence is very rare (only 3 such cases out of 350 polyps of the recto-colon were observed by Duhamel (1964)).

The histology has been amply described. These adenomas are of a particular type, with enormous hyperplasia and inflammation of the chorion; the glandular part is less affected but shows a peculiar irregularity in the size of the glands, some being hypertrophied and some cystic. The epithelial covering may desquamate giving the polyp the appearance of a fleshy bud. Some authors do not accept these as adenomas, but we believe, as Helwig does, that they are adenomas of a special type, for the following reasons: (1) predominance in males, as in adult adenomas and recto-colonic cancers; (2) the frequency of multiple polyps is the same for children and for adults; (3) the existence of forms resembling adult adenomas but only showing slight inflammation initially; and (4) the existence of adult adenomas

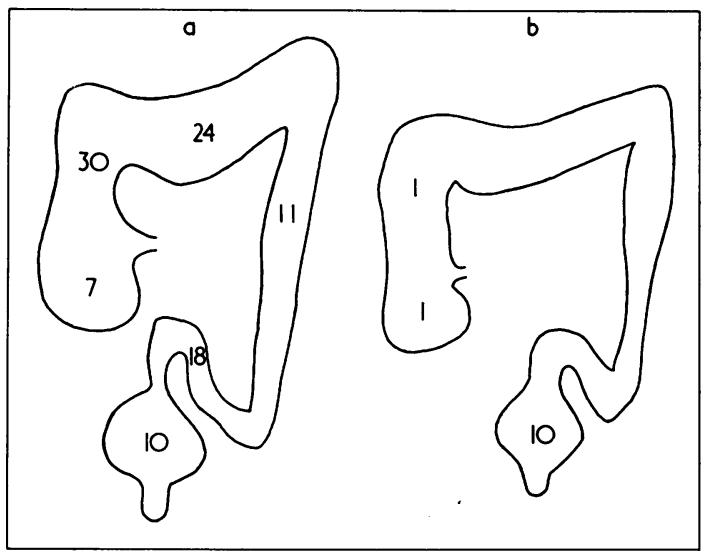

FIG. 1a and 1b.-(a) Distribution of adenomas in adults (after Chapman, 1963; Feyrter, 1931). (b) Distribution of adenomas in children, 449 subjects (after Helwig, 1946). 
TABLE 1

DETAILS OF 'REMOTE' COLONIC POLYPS IN 15 CASES AGED 3 TO 11 YEARS

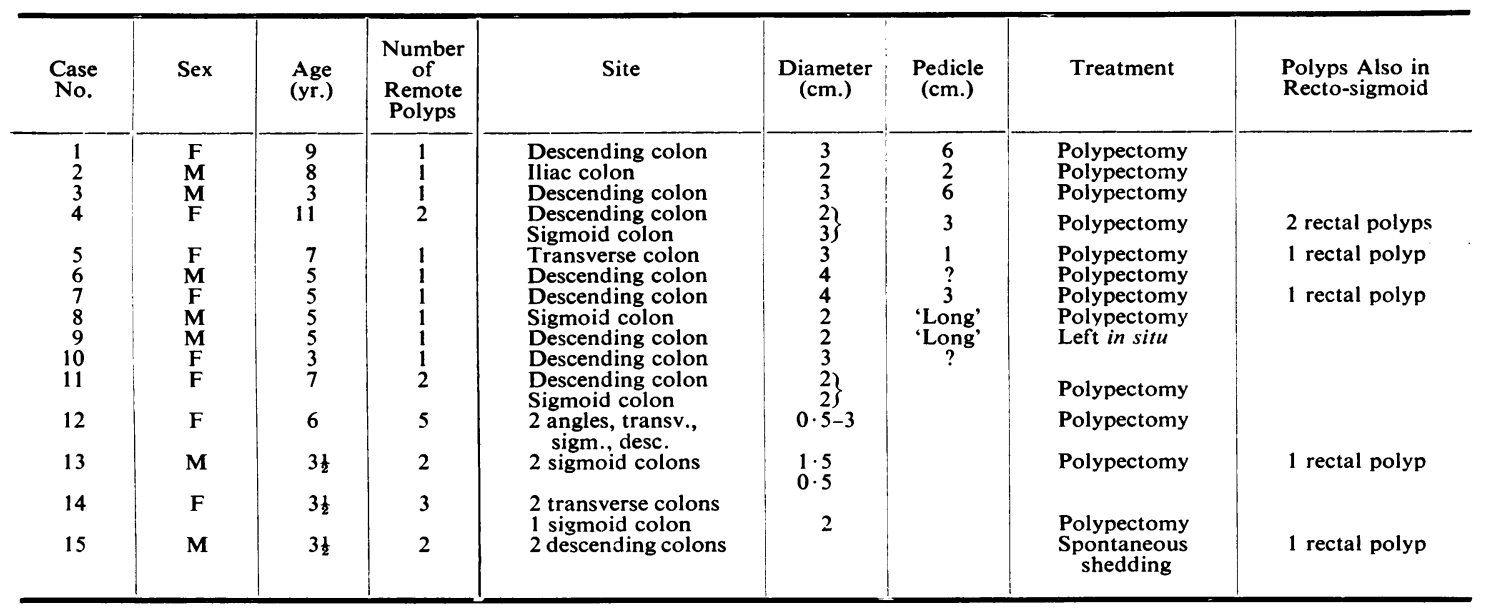

of inflammatory type, histologically resembling those of the child.

In comparing the appearances of remote polyps, we are faced with the problem that they cannot be viewed by sigmoidoscopy, nor are biopsies or endoscopic operations possible.

\section{Clinical Features}

These are summarized in the Table. The average age is between 3 and 5 years as with recto-sigmoidal polyps. Recto-sigmoidal polyps in the child nearly always declare themselves by the appearance of fresh blood in the stools. A polyp of the colon which may bleed slightly, or because of its distance produces only coagulated blood in the faeces, may go unnoticed. A valuable indirect sign of a polyp out of reach of the sigmoidoscope is the presence of traces of fresh blood higher up when a polyp of the rectosigmoid region has already been removed. This is an indication for barium examination of the colon.

\section{Radiography}

All cases require a radiological examination. A special technique is necessary: purge, enema, finely particulate and adhesive barium, moderate filling, pictures taken after evacuation and insufflation, double contrast, patient horizontal and erect. From the study of our $x$-ray material we have noted three characteristic appearances.

(1) Bowel filled or half filled: a transparency (lacuna) more or less circular (14 instances).

(2) Bowel inflated: polyp coated with barium, appearing opaque on a translucent background ( 8 instances).
(3) After evacuation: polyp expanding the collapsed lumen, the 'bead on a string' appearance (3 instances).

The figures show that the 'half-filled' picture more often gives the characteristic appearance, but these results must be completed and confirmed by other technical means. The pedicle has been correctly observed 8 times: 4 times in the 'half-filled' state, once in evacuation, and on 3 occasions during insufflation, each time with the help of compression. Even if the image of the polyp is characteristic, radiological control before operation is absolutely necessary, as it is a check on false images that may be extremely puzzling and even simulate a pedicle.

\section{Anatomy}

The polyp of the colon was single in 9 out of 15 cases. There were multiple (up to 4) polyps in 6 cases. In the collection as a whole there were 5 instances of a remote colonic polyp associated with a recto-sigmoid polyp.

The average size for all remote polyps was from 2 to $4 \mathrm{~cm}$. This shows that even with perfect techniques, small polyps remain unseen. The polyps were situated in 10 instances on the descending colon, in 8 on the iliac colon and high sigmoid, and in 6 on the transverse colon. The predominance of the left colon may be explained by the fact that this portion is of a slight diameter, is tubular and easily compressible, making the contrast between polyp and barium more demonstrable. The pedicle was nearly always long, generally being more than $5 \mathrm{~cm}$.

The histology was the same as that of rectosigmoidal polyps, with inflammatory changes, 
intrapolypoid haemorrhage, some suppurating areas, and surface ulceration.

\section{Spontaneous Evolution}

It appears that evolution proceeds the same way as the recto-sigmoidal polyps, with spontaneous shedding of the polyp due to progressive ischaemia, haemorrhage, and suppuration, leading to a partial or total amputation of the polyp itself or of its pedicle. This seems to happen less often in the colon because here there is no trauma from defaecation or extrusion. The longer life of the polyps in the colon explains why its pedicle is also longer than that of its fellow in the recto-sigmoid. It also implies that views about the prognosis of these polyps must be reserved.

\section{Problems of Recurrence in situ}

Is this spontaneous separation the sign of final healing? Or, on the contrary, is a recurrence on the stump of the pedicle a danger? This is an important question because these polyps cannot be watched by means of endoscopy.

Concerning this spontaneous separation of remote polyps, we have observed a polyp in the upper sigmoid with a long pedicle that was out of sight at a previous examination. Two polyps in the same child (Case 15) were also thought to be behaving in this way. In two other cases (Nos. 9 and 10) in children aged 3 and 5 years, the shedding of the polyps has still not occurred.

If we cannot as yet answer the question for colonic polyps, we have a much larger experience of the recto-sigmoidal polyps, which are of the same nature. We have often observed that after spontaneous detachment of the polyp the pedicle remains, and we have noted its position with accuracy. Two changes may follow. (1) Sometimes the pedicle is absorbed in a few days or a few weeks leaving no trace. (2) More rarely the pedicle remains and grows into a new polyp: such a recurrence may also be seen after an incomplete removal of the polyp by the use of biopsy forceps, insufficient electrocoagulation, or snare. We have tried to reproduce this phenomenon in the following way.

We selected a few cases of low-sited polyps and cut the pedicle at its distal part by means of scissors or biopsy forceps or ligature, deliberately leaving in situ a large segment of the pedicle. A recurrence on the pedicle was observed in several cases in the ensuing months. The end became club-shaped and red, and within a few weeks or months a new polyp had formed.

When the root of the pedicle is resected there is no recurrence.
Histology of the Pedicle. The samples showing the pedicle are scarce, as the polyp is generally removed by the diathermy loop, with fulguration of the pedicle. Lengthwise section of the pedicle is necessary, when three distinct areas may be observed: (1) a proximal area, where glands are compressed one against the other, of small size, regularly shaped, having the same appearance as normal mucous membrane; (2) an intermediate area, with dilated glands with flattened epithelium next to normal glands in a chorion, showing some hypertrophy but little inflammation, and with vascular spaces; (3) a distal area, with all the characteristics of the infantile polyp, but with less marked inflammation, haemorrhage, or suppuration.

We conclude that the propensity for polyp formation resides especially in the middle and distal areas. Resection at the base of the pedicle, or better still in the sound mucous membrane, will not be followed by recurrence, as we have confirmed experimentally. A section in the middle or distal part will favour relapse. Since we do not know where the polypogenous area begins, a fairly deep fulguration is required, while avoiding the risk of a scar. In the recto-sigmoidal area we try to limit the parietal cautery scar to an average of $5 \mathrm{~mm}$. diameter.

\section{Surgical Treatment}

For rectal polyps this presents few problems, though it may be somewhat difficult to regulate which part is to be left to fulguration and which to section when the diathermy loop is used. If coagulation is followed by even slight bleeding, it proves that it has been insufficient, and in that case it must be completed by a fulguration of the remaining stump by means of the ball-shaped electrode or of the diathermic tag. In certain cases it would be simpler to cut the polyp with biopsy tongs and to coagulate the pedicle.

The problems of treating polyps of the colon are more complex. Because of (a) the frequent continuation of bleeding, (b) the persistence in certain cases of rectal polyps from infancy to beyond 15 years (of which we have a few examples), and the impossibility of asserting, from our present standpoint, that a benign adenoma will not in the long run become malignant, we advise surgical intervention without waiting for a spontaneous separation which may turn out to be a false cure. Abdominal incision is sited in relation to the polyp and the latter is then localized by palpation, transparietal traction to note the implantation, and above all endoscopy before removal, using a sterile proctoscope introduced through a colostomy. By inspection above 
and below, not only may the exact site of implantation of the pedicle be located, but other polyps not seen on a radiograph may be found. Segmental resection, which we advise for such polyps in the adult, is not appropriate here; simple polypectomy suffices, but must be performed with particular care, either by excision of the root of the pedicle and its base (colerette d'implantation) or by fulguration of the base of the pedicle. In the latter case, the risk of haemorrhage is small, for in 150 cases in which we removed polyps in the recto-sigmoid by the diathermy noose we only had one instance of haemorrhage following the sloughing of a scar 8 days later.

\section{Summary}

In 400 recto-sigmoidal polyps in children observed at the Hôpital des Enfants-Malades, we have only found 15 colonic polyps. Even with modern radiological techniques small polyps in the colon are rarely detected. Colonic polyps have the same histological pattern as recto-sigmoidal polyps. They are generally spontaneously shed, but not so frequently as recto-sigmoid polyps. This shedding is not proof of healing, as regrowth from the pedicle is possible.

Total destruction is advised, by excision of the root of the pedicle and its base, or by fulguration after colostomy.

\section{REFERENCES}

Chapman, I. (1963). Adenomatous polypi of the large intestine: incidence and distribution. Ann. Surg., 157, 223.

Feyrter, F. (1931). Zur Geschwulstlehre (nach Untersuchungen am menschlichen Darm). I. Polypen und Krebs. Beitr. path. Anat., 86, 664.

Helwig, E B. (1946). Adenomas of the large intestine in children. Amer. J. Dis. Child., 72, 289.

Duhamel J. (1958). Affections Non-Congénitales de l'Anus et du Rectum Chez l'Enfant, p. 260. Masson, Paris.

- (1964). Les polypes recto-sigmoidiens de l'adolescent. Arch. Mal. Appar. dig., 53, 1122. 